\title{
Synchronization of muscular oscillations between two subjects during isometric interaction
}

\author{
Laura V Schaefer (1), Arndt H Torick(1), Hannes Matuschek (2,3), Matthias Holschneider
} $(2,3)$, Frank N Bittmann (1)

(1) Department Regulative Physiology and Prevention, University of Potsdam; (2) Applied and industrial Mathematics, University of Potsdam; (3) Department of Physics and Astronomy, University of Potsdam, Germany

\begin{abstract}
Muscles oscillate with a frequency around $10 \mathrm{~Hz}$. But what happens with myofascial oscillations, if two neuromuscular systems interact? The purpose of this study was to examine this question, initially, on the basis of a case study. Oscillations of the triceps brachii muscles of two subjects were determined through mechanomyography (MMG) during isometric interaction. The MMG-signals were analyzed concerning the interaction of the two subjects with algorithms of nonlinear dynamics. In this case study it could be shown, that the muscles of both neuromuscular systems also oscillate with the known frequency (here $12 \mathrm{~Hz}$ ) during interaction. Furthermore, both subjects were able to adapt their oscillations against each other. This adjustment induced a significant $(\alpha<.05)$ coherent behavior, which was characterized by a phase shifting of approximately $90^{\circ}$. The authors draw the conclusion, that the complementary neuromuscular partners potentially have the ability of mutual synchronization.

Key Words: Mechanomyography, muscle oscillations, synchronization, interpersonal sensorimotor interaction, non-linear analysis, continuous wavelet transform
\end{abstract}

Eur J Trans Myol - Basic Appl Myol 2014; 24 (3): 195-202

Muscles oscillate and generate noises. The auditive detection of contractile skeletal muscles indicates that myofascial structures oscillate. In the 17th century, the Italian theologian, mathematician and physicist Francisco Grimaldi $(* 1618, \quad \dagger 1663)$ already documented, that he caught sounds through covering his ears, which he described as "tremores". ${ }^{1}$ In 1810, the English physician, physicist and chemist W. H. Wollastan concluded his investigations with the result that muscles vibrate at a frequency of about $23 \mathrm{~Hz} .^{2}$ For a long time this issue was rarely regarded in research - only since the 1980s, the area of myofascial oscillation is increasingly considered. Biomechanical investigations confirm the muscular oscillation in kinematics and dynamics - with a stochastic character - of approximately $10 \mathrm{~Hz}^{3-6}$ According to several authors, these oscillations are generated through transversal movements of muscle fibers. ${ }^{7-10}$

Thus, it can be regarded as secured, that oscillation characterizes the functioning of neuromuscular systems. But what happens, if two oscillating neuromuscular systems interact?

Interaction implies, that the dynamics of one system has effects on the other one, while itself is underlying influences of the other systems. ${ }^{11}$ Consequently, interaction means a permanent interdependency of several subsystems, which can be described as a complete system. A dynamic beyond the individual behavior arises. ${ }^{11-12}$

In the 17th century Huygens (1629-1695) already described, that linked periodic oscillating systems synchronize (coupled pendulums). Thus, it can be hypothesized, that this is also the case with two interacting sensorimotor systems.

Until now, examinations of synchronization phenomena are limited to intermuscular coordination in the context of muscle chains, which evaluate the interaction of muscles - only with regard to qualitative muscular activity - e.g. while walking or performing other physical exercises. ${ }^{13-16}$ Investigations of synchronization can also be found regarding to the firing rate of motor units ${ }^{17-18}$ as well as to the relation between brain and muscle activity. ${ }^{19}$

According to current studies, the synchronization of mechanic oscillations of muscles has not been considered yet. However, the oscillation of neuromuscular system cannot be regarded as passive harmonic oscillation, but it is produced actively, shows 


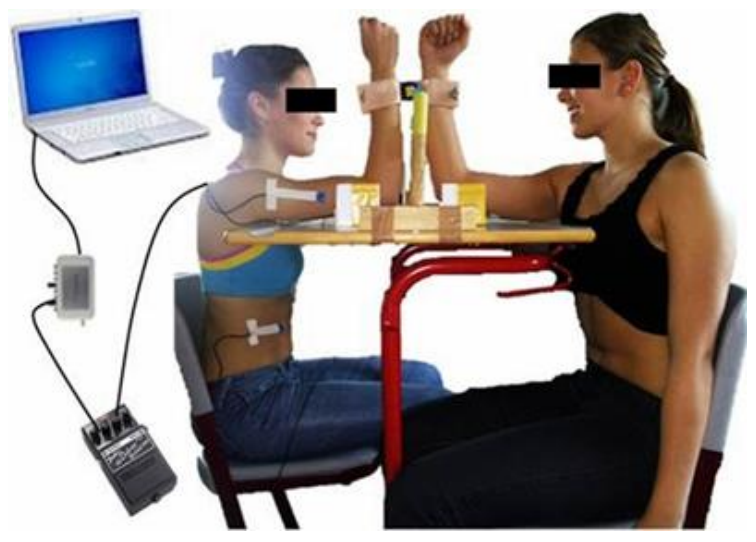

Fig 1. Schematic diagram of interaction. Interaction of two subjects measured by piezoelectric $M M G$-sensors, inter alia, on the triceps brachii muscles. The signals are conducted across an amplifier to an $A / D$-converter and are recorded by the software NI DIAdem 10.2.

a stochastic character, and underlies sensorimotor regulation.

Hence, it is initially unclear, how the two systems will influence each other. The following questions arise:

1. How are the expected muscular oscillations characterized in their frequency shaping?

2. Does adjustment appears relating to the oscillation behavior between the neuromuscular systems, maybe synchronization effects?

3. Is a possibly observed pattern of interaction reproducible?

In this case study we examine interactions of muscular oscillations of two subjects acting against each other.. It is hypothesized that a form of synchronization appears between the interacting neuromuscular systems and we provide mathematical models of tremor and interaction.

\section{Materials and Methods}

In the context of this exploratory study, the myofascial oscillations (MMG) of two interacting persons are analyzed with algorithms of nonlinear dynamics.

\section{Participants}

This case study examines two male sport students at the age of 24 and 23 years, respectively, with a body height of $180 \mathrm{~cm}$ and a body weight of 77 and $69 \mathrm{~kg}$, respectively. Subject A is left handed, subject B right handed. Both subjects practice ball sports: subject B plays handball, subject A exercises soccer and fitness. The subjects had no disorders of the upper limbs, the shoulder belt and the cervical spine at least within the last six months before the measurement.

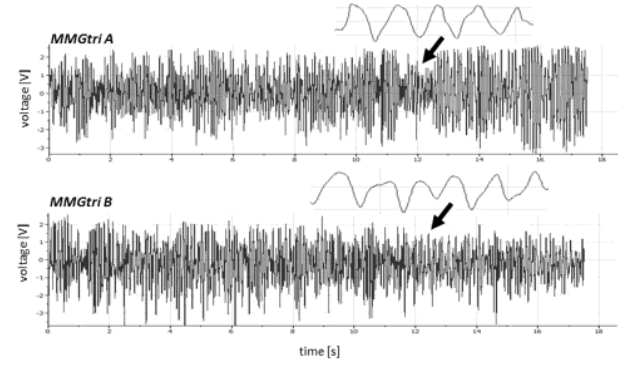

Fig 2. MMG time-series. Amplified raw signals (in volts). MMG-signals of the triceps brachii muscles of subject $A$ and $B$.

\section{Procedure}

The subjects are sitting opposite, but shifted in a way, so that the vertically positioned right forearms are directly towards each other. The angles between leg and trunk, arm and trunk as well as the elbow angle measure $90^{\circ}$ (Fig. 1). The subjects are connected through an interface proximal of the ulnar styloid process. The interface consists of two shells of a thermic deformable polymer material, which is commonly used in rehabilitation technology. The shells are shaped according to the contour of forearms. A strain gauge is located between the shells (model: ML MZ $2000 \mathrm{~N}$ 36) in order to record the reaction force between the subjects. An acceleration sensor (comp.: Biovision) is fixed on the strain gauge to detect the accelerations along the longitudinal acting force vector. Amongst others, the muscle oscillations of the lateral head of the triceps brachii muscle are recorded using piezoelectric MMG-sensors (model: Shadow SH 4001). Due to the transversal muscle oscillation, the lateral positioning of the sensors on the skin above the muscle belly has an influence on the signal. ${ }^{5,9,20,21}$ This has to be considered when fixing the sensors and is beside the difficulty to standardize the contact pressure - one reason for the limited comparability of amplitudes between subjects. The MMG-signals are conducted across an amplifier (Nobels preamp booster pre-1) to an A/D-converter (comp.: Biovision) and subsequently are recorded by the software NI DIAdem 10.2 (National Instruments on Sony Vaio: PCG$61111 \mathrm{M}$, Windows 7). The sampling rate is $1000 \mathrm{~Hz}$. The subjects should adjust an isometric status at $80 \%$ MVC of the weaker subject and maintain this for $15 \mathrm{~s}$. Due to irregularities in maintaining the given force, it partially had to be measured over a longer period (max. $25 \mathrm{~s})$. The suitable isometric phases were partly shorter. The $80 \%$ MVC were determined previously in a maximal test (highest value from two attempts against a solid resistance). The subjects could control the force level via a biofeedback (dial instrument). Four measurements were performed with 2-minute breaks in-between. In this article the analysis includes only the isometric phase. 


\section{Synchronization of muscular oscillations}

Eur J Trans Myol - Basic Appl Myol 2014; 24 (3): 195-202

\section{Data analysis}

First, the raw signals of myofascial oscillations are investigated concerning their frequency using a power spectral density (PSD) and a continuous wavelet transform. To analyze the interaction, the MMG timeserie of the triceps brachii muscle of subject $\mathrm{A}$ is related to the one of subject B. Thereby, the wavelet coherence analysis shows the phase shift between the oscillations and the degree of coherence in the course of time. Through this consideration of both signals, conclusion about potential synchronization phenomena can be drawn.

In addition, the time distances of the local maxima of both MMGs to one another are calculated to illustrate potential synchronization between both neuromuscular systems (Analysis of phase distance).

\section{Continuous Wavelet Transform}

The continuous wavelet transform, i.e., ${ }^{22}$ of a real valued signal $s(t)$ with respect to a mother wavelet $g(t)$ is defined as

$$
\left(\mathcal{W}_{g} s\right)(b, a)=\int_{-\infty}^{\infty} \frac{1}{a} g^{*}\left(\frac{t-b}{a}\right) s(t) d t
$$

Where $\mathrm{g}^{*}$ is the complex conjugated of $\mathrm{g}$. The wavelet transformed $\left(W_{g} s\right)(b, a)$ is a function of the scale $a \in \boldsymbol{R}^{+}$ and dilatation $b \in \boldsymbol{R}$. The scale may be replaced by a frequency, defined as $f=1 / a$ for a convenient interpretation of the resulting transformation. In this article, we utilized the Morlet wavelet $^{22}$ as the mother wavelet, which is defined as

$$
g_{\sigma}(x)=e^{i x} e^{-\frac{x^{2}}{2 \sigma^{2}}}
$$

The wavelet transformed is a time-scale or timefrequency analysis, where the wavelet spectrum $S(b, a)=\left|\left(W_{g} s\right)(b, a)\right|^{2}$ quantifies the energy distribution

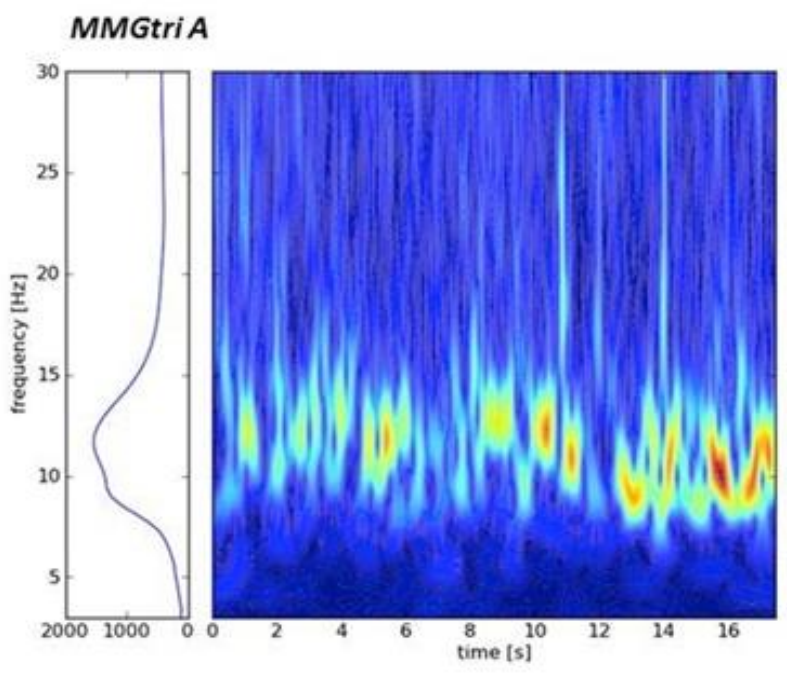

over the time-scale plane. Another explorative feature we used is the instantaneous phase

$$
\phi(b, a)=\arg \left(\left(\mathcal{W}_{g} s\right)(b, a)\right) .
$$

The actual time resolution of the wavelet transformed is governed by the scale (smaller scales have a higher time localization) and the parameter $\sigma$, which is the relative scale/time resolution. For a higher value of $\sigma$ the time resolution drops, while the scale/frequency resolution increases. For the following analyses we use $\sigma=0.5$.

The wavelet transform has proven to be suitable for the analysis of non-stationary processes. ${ }^{23}$ This nonstationarity might be caused by the modification of extrinsic parameters of the experiment as well as unobserved variations of intrinsic process parameters during the time period of the experiment. In contrast to the Fourier spectrum, which has no time-resolution at all, the wavelet transform is capable to quantify changes in the spectral properties of the process over time.

Further spectral estimators can be derived from the wavelet spectrum estimators. The cross wavelet spectrum $^{24,25}$ of two time-series $s_{x}(t)$ and $s_{y}(t)$ can be defined analogously to cross spectrum of the Fourier transform, as the expectation value of the direct product of the wavelet coefficients

$C S_{g}\left[s_{x}, s_{y}\right](b, a)=\left\langle\left(W_{g} s_{x} \cdot W_{g} s_{y}\right)(b, a)\right\rangle$

The averaging $\langle\cdot\rangle$ above can be performed over trials, if a coherent repetition of the experiment is possible. For the experiments described in this article, a coherent repetition of the experiments was not possible. Therefore the averaging must be performed in the time-scale plane using a small window as described in Maraun et al. ${ }^{23}$ From this definition of the cross

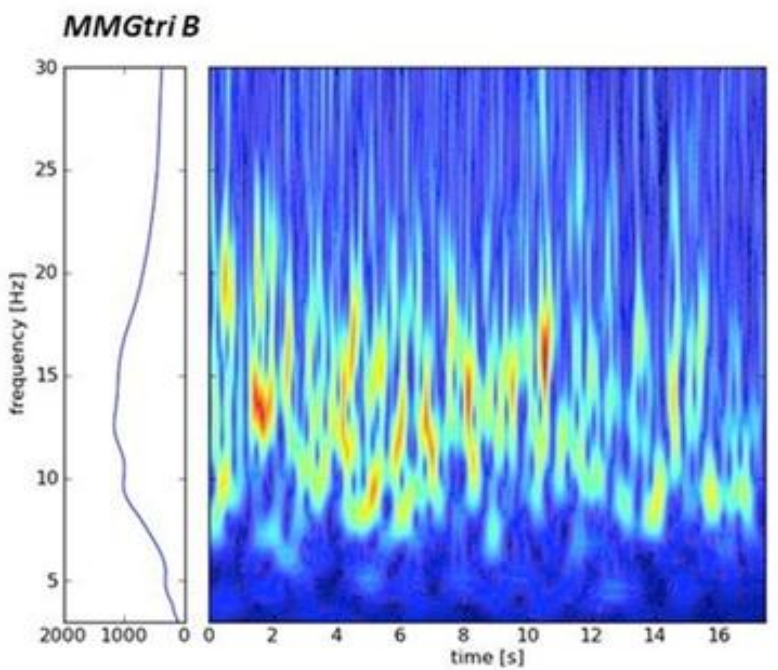

Fig. 3 Wavelet spectra of the MMG-signals. Exemplary contour diagrams of the wavelet spectra of the MMG-signals of subject A (left) and subject B (right). The left panels show the summation of the wavelet spectrum over time, which can be seen as smoothed PSD. 


\section{Synchronization of muscular oscillations}

Eur J Trans Myol - Basic Appl Myol 2014; 24 (3): 195-202

wavelet spectrum estimator the wavelet coherence $\operatorname{Coh}_{g}\left[s_{x}, s_{y}\right]^{24}$ of two time-series can be defined as

$\operatorname{Coh}_{g}\left[s_{x}, s_{y}\right](b, a)=\frac{C S_{g}\left[s_{x}, s_{y}\right](b, a)}{\left|\left\langle W_{g} s_{x}(b, a)\right\rangle\right|\left|\left\langle W_{g} s_{y}(b, a)\right\rangle\right|}$

The modulus of the wavelet coherence $\left|\operatorname{Coh}_{g}\left[s_{x}, s_{y}\right](b, a)\right| \in[0,1]$, quantifies the coherence of the two time-series. Further, the argument of this estimator,

$\arg \left(\operatorname{coh}_{g}\left[s_{x}, s_{y}\right](b, a)\right)=\arg \left(C S_{g}\left[s_{x}, s_{y}\right](b, a)\right) \in[-\pi, \pi)$, reveals the phase difference between the two timeseries at time point $b$ and scale $a$.

The cross wavelet estimator as defined in equation (0.1) and therefore the coherence estimator (equation (0.2)) adheres a variance and a bias. ${ }^{23}$ The variance of the estimator can be reduced on the cost of increasing the bias. Thus, a significance test must be applied to separate spurious coherence patterns in the analysis from significant ones. This is done by selecting candidates based on a point-wise significance test using surrogate data. A detailed description of the algorithm can be found in Maraun et al. $^{23}$ In the following, an alternative approach to the wavelet coherence spectrum is described, which was used additionally to illustrate the synchronization.

\section{Analysis of phase distance}

This algorithm determines the time distance between the local maxima of two signals. It searches for local maxima of signal $\mathrm{A}$ and compares their times with the times of the local maxima of signal B. It determines the time distance to the temporal nearest local maximum of $\mathrm{B}$, irrespective of whether this lies after or before. Afterwards, the time distances between the local maxima are displayed in a bar diagram, in which a positive orientation represents a temporal forward of signal A and a negative one a forward of signal B. The analysis determines if the temporal sequences of the local maxima of both signals towards each other are stochastically distributed or possibly show phases of regular behavior. Thus, a connection relating to the phase shift as well as an indication of synchronization of the MMG-signals can be concluded - but there can only be made propositions of the temporal forward or time lag, but not about the phase difference.

\section{Results}

\section{Description of the separate signals}

Regarding the raw signals of mechanomyography from subject $\mathrm{A}$ and $\mathrm{B}$, they show oscillations (Fig. 2). While the MMG-signal of subject A shows a mean frequency range 8 to $15 \mathrm{~Hz}$, the power spectrum density (PSD) of the signal of subject $\mathrm{B}$ is clearly broader. Frequencies between 5 and $20 \mathrm{~Hz}$ dominate (Fig. 3). A heterogeneous amplitude pattern can be recognized within one MMG time-series, even though the force oscillates around a mean force of $116 \mathrm{~N}(\mathrm{SD}+/-5)$.

These first analysis of separate signals are in good agreement with prior knockledge, in which MMG- signals are oscillating stochastically near by $10 \mathrm{~Hz}$. The authors will report in a succeeding publication that the simultaneously recorded reaction force and acceleration - which are generated by the muscle activity of both subjects - also show this frequency characteristic.

\section{Description of the interaction}

For characterization of the interaction of both subjects, the MMG time-series of the subjects are analyzed using the estimators described above. The blackbordered fields shown in the wavelet coherence spectrum (Fig. 4) represent point-wise significant coherent areas $(\alpha=.05)$. The right scale of the coherence analysis (Fig. 4 bottom) describes the level of coherence. During the whole time period a pointwise significant patch can be recognized in the same frequency band as found in the PSD with frequencies between 6 and $20 \mathrm{~Hz}$. The other smaller contoured patches are considered spurious. Over the entire pointwise significant coherent period of $17 \mathrm{~s}$ a clear phase band between 5 and $18 \mathrm{~Hz}$ appears (Fig. 4 above). The color of the figure represents the phase difference $\Delta \phi$ and can be read off the right scale. In this case, a phase difference of approximately $\Delta \phi \approx \pi / 2$ can be found.

The analyses of the three remaining measurements at $80 \%$ MVC of the same matching show similar results. The estimation of the phase distances (Fig. 5) supports the previous analysis. In particular, the intervals from second three to ten as well as from second 14 to 17 show a non-coincidental distribution of the distances. In total, 145 local maxima of subject $A$ are lying in front of the local maxima of subject B, 76 are lying behind the local maxima of the MMG-signal of subject B. Thus, the MMG of subject $\mathrm{A}$ has an advance in
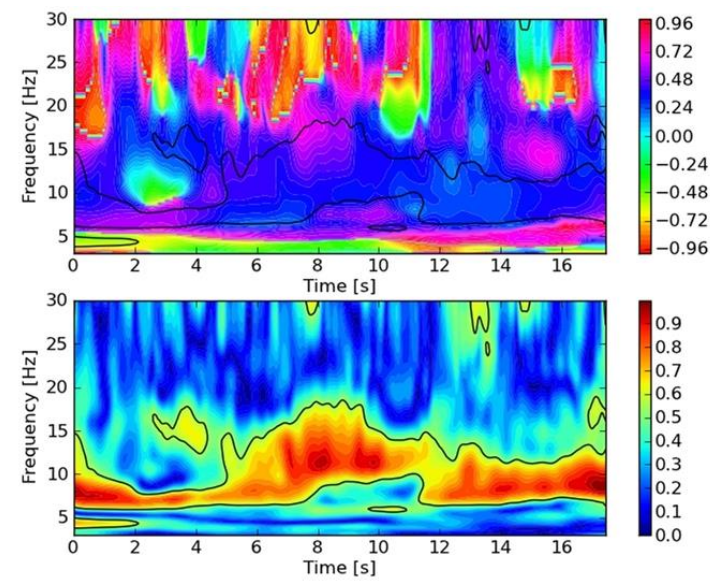

Fig 4. Wavelet coherence spectrum of MMG-signals. Contour diagram of the wavelet coherence spectrum of MMGs of triceps brachii muscles of subject $A$ and $B$. The contour lines show the point-wise significance $(\alpha=.05)$; above: phase difference in fractions of $\pi$; bottom: coherence spectrum. 


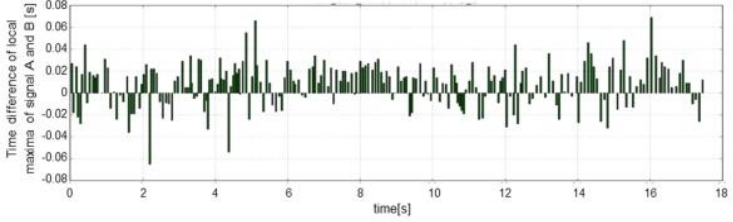

Fig 5. Analysis of phase distance of MMG-signals. Analysis of phase distance of the local maxima of filtered MMG-signals of subject A towards subject B (low pass filter: Butterworth; filter degree: 5, cutoff frequency: $20 \mathrm{~Hz}$ ). Clusters of positive time-lags of local maxima of $M M G$ of subject $A$ in regard to the ones of subject $B$ can be recognized.

relation to the one of subject $\mathrm{B}$. This differs highly significant from coincidental matching $(p<.001)$, in which no such cluster can be found.

Summarizing these analyses of data regarding the interaction, there exists a significant coherent phase shift over the whole time period of the isometric phase of about $\pi / 2$ in a frequency band of approximately 12 $\mathrm{Hz}$.

\section{Discussion}

The findings of this case study concerning the frequency band are - as expected - in agreement with other authors. ${ }^{4}$ This supports the validity of the used measuring and analysis techniques. Nevertheless, we like to emphasize, that the MMG only detects the transversal oscillation related to the muscle belly. This is not necessarily identical to the muscles' force development. But these oscillations are expressions of muscular activity, whose physiological relevance is still unexplained. Whether the superficial measured, mechanic oscillation patterns are corresponding to the actual behavior of the contractile structures and thus of the neuromuscular system, still remain to be investigated.

Even though the mean frequencies of both subjects are approximately $12 \mathrm{~Hz}$, the frequency band of subject B is broader than the one of subject $\mathrm{A}$. This agrees with Beck (2010), who reports from investigations, ${ }^{26}$ in which frequencies between 5 and $25 \mathrm{~Hz}$ were found in biceps brachii muscle. So, the different characteristics of the frequency spectra of the MMG-signals can at least be classified as physiological due to the individuality of biological systems.

The wavelet spectra of the MMG signals, as shown in figure 3 , suggest, that this signal is well described by an autoregressive process of order 2 (AR(2) process). A realization of this time-discrete and stationary stochastic process can be considered as filtered white noise, i.e., $\mathrm{x}_{\mathrm{t}}=\mathrm{ax}_{\mathrm{t}-1}+\mathrm{bx}_{\mathrm{t}-2}+\varepsilon_{\mathrm{t}}$, where $\varepsilon \mathrm{t} \sim \mathrm{N}\left(0, \sigma^{2}\right)$ is identical and independent normal distributed (i.i.d.). With $\mathrm{a}=0.5, \mathrm{~b}=-0.5$ and $\sigma=1$ the $\mathrm{AR}(2)$ process has

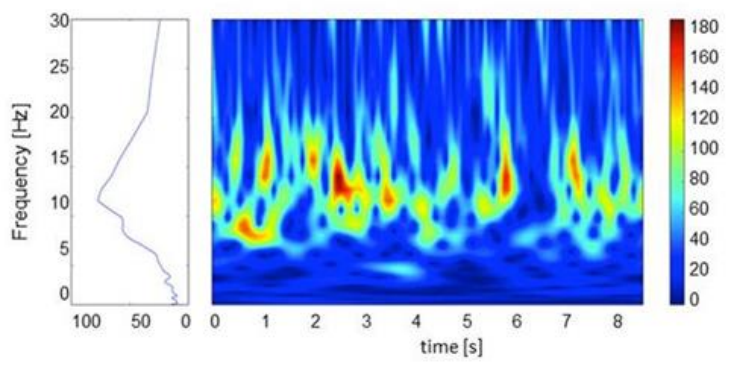

Fig 6. Wavelet spectrum of a realization of an $A R(2)$ process. The process parameters are $a_{x}=0.5$ and $b_{x}=-0.5$. It represents a stochastic process which is in good agreement with the wavelet spectra of the MMGs as shown in figure 3 .

a similar power spectrum as the measured physiological tremor. Figure 6 shows that the wavelet spectrum of this realization is in a good agreement with the wavelet spectrum of the MMGs (compare figure 3). Therefore we can conclude that the myofascial oscillation is well described by an autoregressive process of second order, hence a stochastic process.

It therefore appears that - also during interaction both neuromuscular subsystems oscillate. The result that muscular oscillations of the subjects are coherent in the relevant frequency range supports the assumption, that both neuromuscular systems are adapting themselves to each other, they are able to synchronize. This means that both subsystems of two complementary partners are able to affiliate with each other. Such a complementary system would make high demands on sensorimotor functionality of involved neuromuscular systems. It can be expected, that an undisturbed interaction can only be possible in healthy complementary partners. Further studies are reserved to investigate, whether disturbance of participating persons (disorder, fatigue, disease) is reflected in quality of such an interaction.

This in turn raises the question about the influence of the oscillating (skin-) surface on the measured MMG, from which we assume that it represents a part of the muscular function. In the subsequent discussion we adopt the hypothesis, that the MMG represents an oscillating of force development. This would mean that both systems synchronize also regarding their force progression.

The wavelet coherence analysis suggests that the MMG-signals of both subjects show a phase shift of approximately $\pi / 2$ in the predominant frequency band. According to this, the two systems are obviously not striving after an in-phase synchronization, thus a phase shift of $0^{\circ}$. This would mean that both partners would generate their maximum force exactly at the same time. Such a strategy of interaction would ideally provide mutual even forces at each moment, which 
a
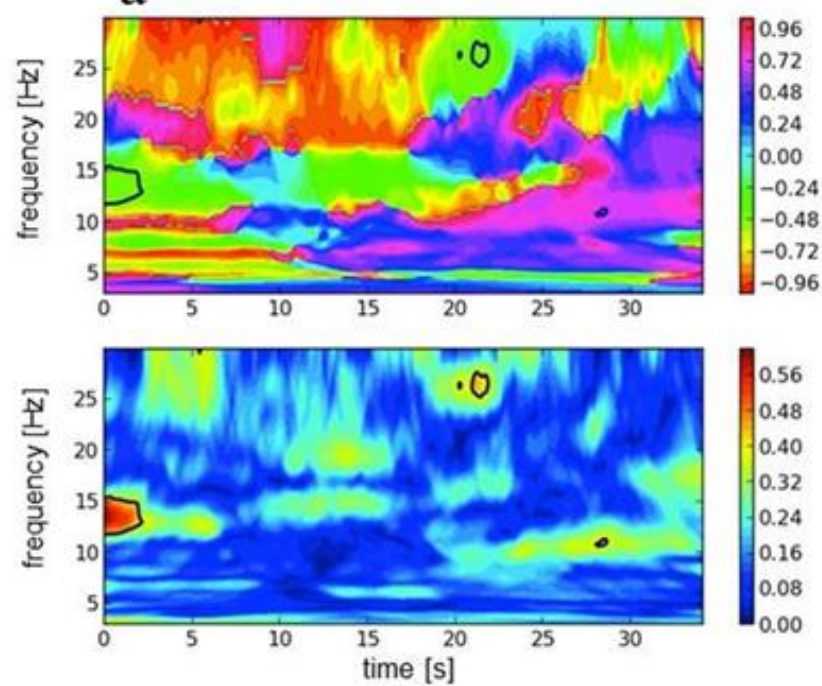

b
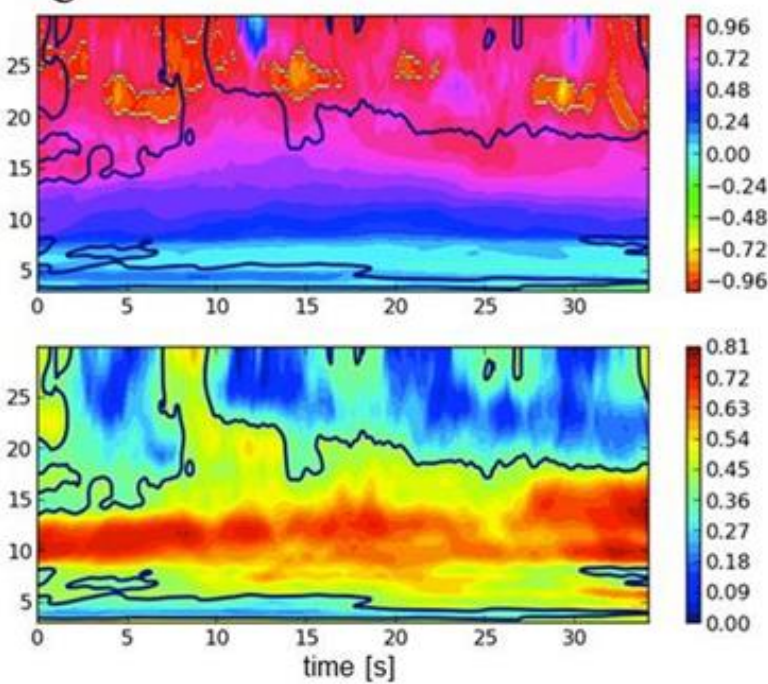

Fig. 7 Wavelet coherence analysis of uncoupled (a) and coupled (b) AR(2) processes. The process parameters are $a_{x}=a_{y}=0.5$ and $b_{x}=b_{y}=-0.5$. Therefore, the two AR(2) processes are identical, hence with an identical power spectra. Wavelet coherence analysis of two uncoupled AR(2) processes. Only spurious coherent behavior is detected as point-wise significant. The two time-series can be considered as incoherent over the complete interval. Asymmetric coupling of $x_{t}$ and $y_{t}$, where $x_{t}$ drives $y_{t}$. A large area of point-wise significant coherence is detected over the complete time interval around $10 \mathrm{~Hz}$. The phase difference between the two processes at that frequency band is approximately $\pi / 2$.

would then be continuously cancelled to zero. A stable or nearby zero crimping force would mean no or only minimal accelerations of the complete system, thus highest stability - a principle used for the lowvibration boxer engine. However, both systems also do not strive after the reversed case - an anti-phase synchronization. This would be given with a $180^{\circ}$ displacement and characterized by a strongly oscillating resulting force, which in turn would generate high accelerations. The found phase shift of around $90^{\circ}$ suggests, that neither of both strategies is "chosen" by the partners, but an intermediate alternative. Furthermore, this means that one partner precedes of about a quarter-cycle with regard to the other partner. Hence, the hypothesis can be proposed that a leader-follower-mode arises during isometric interaction of two subjects. This assumption is supported by the following theoretical description for the MMG time-series.

In order to characterize the MMG signals of interacting persons under the assumption that the physiological tremor in an uncoupled setting is well described by an autoregressive process of 2 nd order (AR(2) process) (see above), two coupled $\operatorname{AR}(2)$ processes $\left(x_{t}\right.$ and $\left.y_{t}\right)$ might be considered:

$$
\begin{aligned}
& x_{t}=a_{x} x_{t-1}+b_{x} x_{t-2}+c_{y} y_{t-1}+\epsilon_{x, t} \\
& y_{t}=a_{y} y_{t-1}+b_{y} y_{t-2}+c_{x} x_{t-1}+\epsilon_{y, t}
\end{aligned}
$$

where $\epsilon_{x, t}$ and $\epsilon_{y, t}$ are identical and independent normal distributed with zero-mean and variance $\sigma^{2}$. The coefficients $c_{y}, c_{x}$ specify the coupling strength of $y$ towards $\mathrm{x}$ and $\mathrm{x}$ towards $\mathrm{y}$, respectively. If $0=c_{y}=c_{x}$, the two processes are uncoupled, thus independent $\mathrm{AR}(2)$ processes. In this case no coherence between realizations of these processes is expected (see figure 7a). In case of $0=c_{y} \neq c_{x}$, the process $x_{t}$ is coupled to $y_{t}$ but not $y_{t}$ to $x_{t}$. Therefore $x_{t}$ can be considered to drive $y_{t}$. Thereby, a coherent behavior of $x_{t}$ and $y_{t}$ can be expected. In analogy to driven harmonic oscillators, the phase difference is $\Delta \varphi \approx \pi / 2$, as shown in figure $7 \mathrm{~b}$.

The wavelet spectra and coherence of the coupled $\mathrm{AR}(2)$ processes are in a good qualitative agreement with the spectra and coherence of the MMG signals (compare figures 4 and $7 \mathrm{~b}$ ). Thus, we can conclude that a) the MMG signals are well described by coupled $\mathrm{AR}(2)$ processes and b) the processes are asymmetrically coupled. This is a strong indication for a leader-follower setting in the experiment described in this article.

This implies obligatory, that one partner hurries ahead, the other follows. De facto, the curve local maxima of subject A are found mostly in front of those of subject B. This also supports the hypothesis that a leaderfollower-constellation appears within such a dynamic oscillating equilibrium. In this grouping, subject A potentially corresponds to the leader-function because his signal is hurrying ahead. Therefore it is possible, 


\section{Synchronization of muscular oscillations}

Eur J Trans Myol - Basic Appl Myol 2014; 24 (3): 195-202

that he is dominating the movement and subject $\mathrm{B}$ is reacting to its input - as described by the coupled forced $\mathrm{AR}(2)$ process. Eventually this mode is responsible for the broader frequency spectrum of subject B compared to the one of subject A. This hypothesis has to be proven in further investigations. The described phenomenon for this subject pair was reproducible over all four measurements. The present inspection of further measurements has shown, that also in other pairings comparable phenomena can be identified. Thus, the hypothesis made at the beginning can at least be accepted in principle: Two neuromuscular systems are able to synchronize their myofascial oscillations during interaction. For this reason it can be expected, that the complete system is exposed to oscillating accelerations. This problem will be considered in a succeeding publication. Which advantages such an approach will implicate, can only be guessed. Obviously, also coupled muscle systems strive after an oscillating force development, like they also do solitary. It can therefore be concluded, that oscillations could be necessary for intact muscle function in any circumstance.

In conclusion, muscles do not only oscillate, but also, as this case study suggests, the interacting muscular systems are able to synchronize their oscillation behavior.The results propose that a permanent sensorimotor regulation of the systems takes place, they adapt to each other, but they allow certain variance. For further investigations in particular the leader-follower-constellation seems to be relevant. The authors will publish how this behavior will affect the generated forces and thus the accelerations. For followup consideration of interaction between regulating neuromuscular systems the subsequent questions arise:

1. Is the interpersonal neuromuscular synchronization an individual case or can it be recovered recurring as a stable phenomenon?

2. Does one complementary partner undertakes the task of the leader-function during isometric interaction?

3. Which consequences and characteristics are possibly arising for the follower-mode?

4. Is the potential existing follower-mode characterized by a broader frequency spectrum than the leader-mode?

5. Which forces arise from both subsystems as well as between them and how can they be described?

In the course of upcoming investigations, the analyses have to be further optimized. In this regard, the focus is especially on the quantitative representation of synchronization and coherence including significance testing, respectively, as well as on further algorithms for the description of the leader-follower-constellation. The interpersonal synchronization of muscular oscillations establishes an innovative area of research as, incidentally, also the intrapersonal synchronization.
The analysis of oscillation and interaction of neuromuscular systems could contribute to an understanding of physiology, pathophysiology and biomechanics of musculoskeletal system. It is not predictable at the moment which perspectives will be open in the future.

\section{Corresponding Author}

Laura Schaefer, Department of sports and health sciences, Unit Regulative Physiology and Prevention, University of Potsdam Karl-Liebknecht-Str. 24-25, 14476 Potsdam/Golm, Germany. Phone: +49 331 9772898

E-mail: 1schaefe@uni-potsdam.de

\section{References}

1. Grimaldi F. Physiomathesis de Lumine: coloribus, et iride. Fr. Io. Vincentius Paulinus de Garexio Mag. Inquisitor Generalis Bonon, 1665. [http://books.google.de/books?hl=de\&lr=\&id=sB 86AQAAIAAJ $\&$ oi $=$ fnd $\&$ pg $=$ PA $1 \& d q=$ Grimaldi +Physico+Mathesis+de+Lumina\&ots=0e8Gd_zg B1\&sig=bEhj7rT8PWgYQWVH688p2DYUU54 \#v=twopage \&q=Grimaldi\%20Physico\%20Mathe sis\%20de\%20Lumina\&f=false

2. Wollaston WH. The Croonian Lecture. Part I: On the Duration of Muscular Action. Philos Trans R Soc Lond B Biol Sci 1810;100: 1-5.

3. Horsley V, Schäfer EA. Experiments on the character of the muscular contractions which are evoked by excitation of the various parts of the motor tract. J Phys 1886 7: 96-110.

4. McAuley JH, Marsden CD. Physiological and pathological tremors and rhythmic central motor control. Brain 2000;123:1545-67.

5. Beck T. Applications of mechanomyography for examining muscle function. Editor Travis W. Beck, 2010.

6. Torick A. Mechanische Oszillationen der Mm. vastus lateralis et rectus femoris bei isometrischer Kontraktion. Eine mechanomyografische Analyse. Diploma thesis. University of Potsdam, Department of Regulative Physiology and Prevention, 2012.

7. Barry DT. Acoustic signals from frog skeletal muscle. Biophys J 1987;51:769-73.

8. Barry DT, Cole NM. Fluid mechanics of muscle vibrations. Biophys J 1988;53:899-905.

9. Cescon C, Madeleine P, Farina D. Longitudinal and transverse propagation of surface mechanomyographic waves genereated by single motor unit activity. Med Biol Eng Comput 2008; 46: 871-7.

10. Frangioni JV, Kwan-Gett TS, Dobrunz LE, McMahon TA. The mechanism of low-frequency sound production in muscle. Biophy $\mathrm{J}$ 1987;51:775-83. 
Synchronization of muscular oscillations

Eur J Trans Myol - Basic Appl Myol 2014; 24 (3): 195-202

11. Bossel H. Systeme, Dynamik, Simulation: Modellbildung, Analyse und Simulation komplexer Systeme. Norderstedt, Books on Demand GmbH, 2004.

12. Pikovsky A, Rosenblum M, Kurths J (eds): Synchronization. A Universal Concept in Nonlinear Sciences. Cambridge: Cambridge University Press, 2001.

13. Hopf HC, Hufschmidt H-J. Koordination benachbarter Muskeln bei einfachen Willkürbewegungen. Deutsche Zeitschrift für Nervenheilkunde 1963;185:191-202.

14. Marées H de (ed): Sportphysiologie. Köln, Sport und Buch Strauß, 2003.

15. Tittel K (ed): Beschreibende und funktionelle Anatomie des Menschen (12. neu bearb. Auflage). Jena, Stuttgart, Fischer, 1994.

16. Zajac FE: Understanding muscle coordination of human leg with dynamical simulations. J Biom 2002;35:1011-8.

17. Hoffmann P. Über ein Synchronisationsphänomen der Innervation bei willkürlicher Haltung. Deutsche Zeitschrift für Nervenheilkunde 1959;180:84-93.

18. Weytjens JLF, Steenberghe D van. The Effects of motor Unit Synchronization on the Power Spectrum of the Electromyogram. Biological Cybernetics, 1984;51:71-7.

19. Milner-Brown HS, Stein RB, Lee RG. Synchronization of human motor units: possible roles of exercise and supraspinal reflexes.
Electroencephalogr Clin Neurophysiol 1975;38:245-54.

20. Kaczmarek P, Celichowski J, Kasiński A. Experimentally verified model of mechanomyograms recorded during single motor unit contractions. $\mathrm{J}$ Electromyogr Kinesiol 2005; 15: 617-30.

21. Farina D, Li X, Madeleine P. Motor unit acceleration maps and interference mechanomyographic distribution. J Biomech 2008; 41: 2843-2849.

22. Holschneider M. Wavelets: An Analysis Tool. Oxford: Oxford Science Publications; 1995.

23. Maraun D, Kurths J, Holschneider $M$. Nonstationary Gaussian processes in wavelet domain: Synthesis, estimation and significance testing. Phys Rev E Stat Nonlin Soft Matter Phys 2007;75:1-14.

24. Schaefli B, Maraun D, Holschneider M. What drives high flow events in the Swiss Alps? Recent developments in wavelet spectral analysis and their application to hydrology. Advances in Water Resources 2007;30:2511-25.

25. Hudgins L, Friehe CA, Mayer ME. Wavelet transforms and atmopsheric turbulence. Phys Rev Lett 1993;71:3279-83.

26. Mealing D, Long G, McCarthy PW. Vibromyographic recording from human muscles with known fibre composition differences. $\mathrm{Br} \mathbf{J}$ Sports Med 1996;30:27-31. 